\title{
BMJ Open Value of an outpatient transition clinic for young people with inflammatory bowel disease: a mixed- methods evaluation
}

\author{
Jane N T Sattoe (D) , ${ }^{1}$ Mariëlle A C Peeters, ${ }^{1,2}$ Jannie Haitsma, ${ }^{1}$ \\ AnneLoes van Staa, ${ }^{1,2}$ Victorien M Wolters, ${ }^{3}$ Johanna C Escher ${ }^{4}$
}

To cite: Sattoe JNT,

Peeters MAC, Haitsma J, et al. Value of an outpatient transition clinic for young people with inflammatory bowel disease: a mixedmethods evaluation. BMJ Open 2020;10:e033535. doi:10.1136/ bmjopen-2019-033535

- Prepublication history for this paper is available online. To view these files, please visit the journal online (http://dx.doi. org/10.1136/bmjopen-2019033535).

Received 12 August 2019 Revised 06 November 2019 Accepted 10 December 2019

Check for updates

(C) Author(s) (or their employer(s)) 2020. Re-use permitted under CC BY-NC. No commercial re-use. See rights and permissions. Published by BMJ.

${ }^{1}$ Research Centre Innovations in Care, Rotterdam University of Applied Sciences, Rotterdam, The Netherlands

${ }^{2}$ Erasmus School of Health

Policy \& Management, Erasmus University Rotterdam, Rotterdam, The Netherlands ${ }^{3}$ Department of Pediatric Gastroenterology, University Medical Center Utrecht, Utrecht, The Netherlands

${ }^{4}$ Department of Pediatric Gastroenterology, Erasmus Medical Centre - Sophia Children's Hospital, Rotterdam, The Netherlands

Correspondence to

Dr AnneLoes van Staa;

vanstaa@eshpm.eur.nl

\section{ABSTRACT}

Objective Developing and evaluating effective transition interventions for young people (16-25 years) with inflammatory bowel disease (IBD) is a high priority. While transition clinics (TCs) have been recommended, little is known about their operating structures and outcomes. This study aimed to gain insight into the value of a TC compared with direct handover care.

Design Controlled mixed-methods evaluation of process outcomes, clinical outcomes and patient-reported outcomes.

Setting Two outpatient IBD clinics in the Netherlands. Participants Data collection included: semistructured interviews with professionals $(\mathrm{n}=8)$, observations during consultations with young people ( $5 \times 4$ hours), medical chart reviews of patients transferred 2 to 4 years prior to data collection ( $n=56$ in TC group; $n=54$ in control group) and patient questionnaires ( $n=14$ in TC group; $n=19$ in control group).

Outcomes Data were collected on service structures and daily routines of the TC, experienced barriers, facilitators and benefits, healthcare use, clinical outcomes, selfmanagement outcomes and experiences and satisfaction of young people with IBD.

Results At the TC, multidisciplinary team meetings and alignment of care between paediatric and adult care providers were standard practice. Non-medical topics received more attention during consultations with young people at the TC. Barriers experienced by professionals were time restrictions, planning difficulties, limited involvement of adult care providers and insufficient financial coverage. Facilitators experienced were high professional motivation and a high case load. Over the year before transfer, young people at the TC had more planned consultations ( $p=0.015$, Cohen's $d=0.47$ ). They showed a positive trend in better transfer experiences and more satisfaction. Those in direct handover care more often experienced a relapse before transfer $(p=0.003)$ and had more missed consultations ( $p=0.034$, Cohen's $d=-0.43$ ) after transfer.

Conclusion A TC offer opportunities to improve transitional care, but organisational and financial barriers need to be addressed before guidelines and consensus statements in healthcare policy and daily practice can be effectively implemented.
Strengths and limitations of this study

- This study is unique in its design, since it is the first transition clinic (TC) evaluation study to include a control group and similar controls, while employing a reasonably long monitoring period, covering the 2 years before transfer and the 2 years after transfer.

- The mixed-methods design is a strength, because it provided a more comprehensive interpretation of the results, for instance about the organisation and interventions employed in the current TC model.

- A limitation of the study is the insufficient statistical power to demonstrate significant differences in patient-reported outcomes between settings, since the response rate on the questionnaires was low $(37 \%-46 \%)$.

- In addition, patients transferred to other hospitals could not be included in the post-transfer measurements, despite repeated efforts to collect these.

\section{INTRODUCTION}

In recent years, much attention has been given to the development, implementation and evaluation of transition interventions for young people with inflammatory bowel disease (IBD). Two years ago, a national guideline on transition of young people with chronic digestive diseases, including IBD, was developed in the UK. ${ }^{1}$ The need to improve care for these young people has been repeatedly emphasised, ${ }^{2}$ for instance in a topical review on transitional care for young people with IBD by the European Crohn's and Colitis Organisation $^{3}$ and in a position statement by the Italian Society of Gastroenterology. ${ }^{4}$

IBD is a collective term for ulcerative colitis, Crohn's disease and IBD unclassified. While growing up, young people with IBD must learn to manage a very demanding and complex disease. For a correct diagnosis and treatment efficacy, repeat endoscopy is necessary as well as often lifelong treatment with a combination of medication and possibly 
surgery. Treatment is aimed at controlling inflammation and preventing exacerbations. ${ }^{5}$ In adolescents, IBD is more severe than in adults, ${ }^{6}$ showing an unpredictable pattern that is hard to adjust treatment and daily life to.

Next to physical complaints such as abdominal pain, fatigue and side effects of medication, IBD is associated with psychological and social problems in young people. ${ }^{78}$ Many young people with IBD must deal with stress, fear, depressive symptoms and school absenteeism more than their healthy age-mates. ${ }^{9}$ As a result of all this, healthrelated quality of life is compromised. ${ }^{5}$ These young people are especially vulnerable when going through the overall transition from childhood to adulthood and being transferred from paediatric to adult healthcare. Research shows that during these transitions, young people may resist strict treatment regimens leading to non-adherence to medication and drop-out of healthcare. Consequently, they are at risk for complications and worsening health. ${ }^{10}$ Indeed, adolescents with IBD are reported to have more missed consultations and more hospital admissions than adults with IBD. ${ }^{6}$

To support young people with IBD during their transition from paediatric to adult care and to address both the physical and psychosocial burden of their disease, a profound recommendation made is to implement transition clinics (TCs). ${ }^{361112}$ Although there is no common definition of a TC, the core principle is that professionals from paediatric care and adult care jointly deliver outpatient care. ${ }^{21314}$ While TCs are advocated as best practice in transitional care, clinical practice in this area is just emerging and there is still little understanding about service structures, experiences and outcomes of TCs. This is especially true for transitional care for young people with IBD. ${ }^{215}$ Also, it is important for evaluation studies to consider both patient satisfaction and clinical outcomes. ${ }^{2}$ This study aimed to gain insight into the value of a TC compared with direct handover care for young people with IBD by employing a mixed-methods approach. This approach gave room for new insights into outcomes of a TC for young people with IBD while considering its context.

\section{MATERIALS AND METHODS \\ Study design}

The study included a controlled mixed-methods evaluation of process, clinical and patient-reported outcomes. The TC setting was compared with direct handover care for young people with IBD on service structures and daily routines, healthcare use, clinical outcomes, selfmanagement outcomes and experiences and satisfaction with the transfer. Health professionals' experienced barriers and facilitators for the functioning of the TC and perceived benefits were also studied. The study was set in two clinics in university medical centres in the Netherlands, of which one was a TC. The full study protocol has been published before. ${ }^{16}$ The SQUIRE (Standards for
Quality Improvement Reporting Excellence) reporting guidelines were used.

\section{Data collection and study sample}

Service structures and daily routines were studied with semistructured interviews with healthcare professionals and observations during consultations with young people. The interviews also served to gain insight into experienced barriers, facilitators and benefits of the TC. Themes addressed during the interviews were based on relevant literature. These included the validated 'You're welcome' quality criteria that determine whether a clinic can be typified as young people-friendly, ${ }^{17}$ the "Mind the Gap' tool that is used to measure the difference between ideal and experienced transitional care, ${ }^{18}$ and known experiences of young adults, parents and professionals. ${ }^{10-23}$ Examples of topics are reasons for (not) setting up a TC, usefulness of the TC, barriers and facilitators, coordination of the TC, structure and content of transitional care and changes over time, composition of the healthcare team, use of specific interventions and the perceived value of the TC for young people, their parents and healthcare professionals. Interviews lasted for 45 to $60 \mathrm{~min}$ per respondent. Non-participant observations of outpatient consultations were conducted at both settings. In the control setting, regular consultations were observed. Researchers took field notes and wrote down their findings in narratives. The observers focused on coordination of the transition process, content of consultations, interaction between involved parties and use of transition interventions. Professionals from the TC were interviewed between May and July 2014 and those from the control setting between February and April 2015. Observations in the TC setting took place in the period May-July 2014, and in the control setting this was the case for the period June-August 2015.

All young people who had been transferred between 2010 and 2013 (2-4 years before data collection) were included in a chart review and were asked to fill out a survey. Those with severe intellectual disabilities or known psychiatric problems were excluded. Healthcare use and clinical outcomes were assessed through patient chart reviews, at four points in time: the second year before transfer $=\mathrm{T} 1$, the year before transfer $=\mathrm{T} 2$, the year after transfer=T3 and the second year after transfer=T4. Self-management outcomes, experiences and satisfaction were studied with online surveys among the young people, filled out between March and October 2015.

Since fully attending scheduled visits in adult care is an indicator for a successful transition, ${ }^{24}$ the primary outcome for the chart review was the number of missed consultations after transfer. The sample size calculation was based on this outcome and indicated we needed a sample size of 72 or more in each group. ${ }^{16}$ These sample sizes could not be achieved, however, since the populations that met the inclusion criteria at both sites numbered less than 72 per group. At both sites, all young people that met the inclusion criteria were included. The 
Table 1 Quantitative data collection method, method description and outcome measures

\begin{tabular}{|c|c|c|}
\hline Method & Description & Themes addressed or outcome measures \\
\hline Chart review & $\begin{array}{l}\text { Of each outpatient department, all } \\
\text { charts were reviewed of patients who } \\
\text { transferred to adult care 2-4 years } \\
\text { prior to data collection. Data from } \\
\text { chart reviews were collected for four } \\
\text { moments over time: second year before } \\
\text { transfer (T1), year before transfer (T2), } \\
\text { year after transfer (T3) and second } \\
\text { year after transfer (T4). T3 and T4 data } \\
\text { were only available for young people } \\
\text { who transferred to an adult department } \\
\text { within the same hospital. Patients with } \\
\text { severe intellectual disabilities or known } \\
\text { psychiatric problems were excluded. }\end{array}$ & $\begin{array}{l}\text { Background characteristics: Date of birth, date of diagnosis, gender, } \\
\text { comorbidity. } \\
\text { Transfer: Date of transfer, current healthcare provider (department to } \\
\text { which young person transferred is recorded/known). } \\
\text { Medical follow-up: No-show at first appointment in adult care, } \\
\text { number of scheduled/missed consultations, number of planned and } \\
\text { unplanned hospitalisations, emergency department visits. } \\
\text { Clinical outcomes: } \\
\text { Use of medications, in four categories: } 1=\text { aminosalicylates, } \\
2=\text { corticosteroids; } 3=\text { immunomodulators; } 4=\text { biologicals/biosimilars. } \\
\text { Disease activity during transfer, measured by global physician } \\
\text { assessment in four categories (remission, mild, moderate and } \\
\text { severe) dichotomised as being active (moderate or severe) or } \\
\text { not (remission or mild), or measured by the Paediatric Ulcerative } \\
\text { Colitis Activity Index (PUCAI)/weighted Paediatric Crohn's Disease } \\
\text { Activity Index (wPCDAI), where a score of } 10 \text { or higher indicated } \\
\text { active disease during transfer. } \\
\text { Whether young people had a relapse in the year before transfer } \\
\text { (T2) or after transfer (T3). }\end{array}$ \\
\hline Survey & $\begin{array}{l}\text { An invitation for a digital survey was } \\
\text { sent to all patients whose charts were } \\
\text { reviewed. }\end{array}$ & $\begin{array}{l}\text { Background characteristics: Educational level, type of education, } \\
\text { disability benefits. } \\
\text { Healthcare-related outcomes: } \\
\text { Transfer experiences measured with the validated 18-item On Your } \\
\text { Own Feet Transition Experience Scale with 5-point Likert scales. }{ }^{35} \text {. }^{\text {. }} \text { Self-reported satisfaction with transition on a 1-10 scale. } \\
\text { Perceived patient-centredness measured with a subscale of the } \\
\text { American Consumer Assessment of Health Plan Surveys (validated } \\
\text { 5-item scale with 4-point Likert scales. }{ }^{36} \\
\text { Self-management-related outcomes: } \\
\text { Self-management measured with the validated } 12 \text {-item Partners in } \\
\text { Health Scale with 9-point Likert scales. }{ }^{37} 38 \\
\text { Independence during consultations measured as self-reported } \\
\text { independence on a 1-10 Visual Analogue Scale. } \\
\text { Self-efficacy measured with the validated } 16 \text {-item On Your Own } \\
\text { Feet Self-Efficacy Scale with 4-point Likert scales. }{ }^{39} \\
\text { Adherence to treatment measured with the validated 5-item } \\
\text { Medication Adherence Rating Scale with 5-point Likert scales. }{ }^{40} \\
\text { Health-related quality of life measured with the validated } 23 \text {-item } \\
\text { Paediatric Quality of Life Inventory Young Adult with 5-point Likert } \\
\text { scales. }{ }^{41}\end{array}$ \\
\hline
\end{tabular}

primary outcome for the survey was transfer experiences. Details on the quantitative data collection are presented in table 1 .

\section{Data analyses}

Interviews were audio taped and transcribed verbatim. Interview transcripts and observation narratives were coded thematically by two researchers (JNTS and MACP) independently. The research applied the framework approach, whereby themes addressed during the interviews and observations were leading in the coding process. If applicable, subthemes were derived from the data. ATLAS.ti 7.0 was used for qualitative data analysis.

Independent samples t-tests and $\chi^{2}$ tests served to test differences between the intervention and control groups in chart review and survey outcomes, and effect sizes were calculated. Cases with missing data were omitted. IBM SPSS Statistics 25 was used for quantitative data analysis.

\section{Ethical considerations}

Young people and, if applicable, their parents received an information letter from the doctor in charge of treatment. They provided written informed consent per study part. Data were processed anonymously and pseudonyms were used in the interview transcripts and observation narratives.

\section{Patient and public involvement}

Patients or the public were not involved in the design, conduct, reporting or dissemination of our research. 
Table 2 Study response per data collection method per setting

\begin{tabular}{|c|c|c|}
\hline Data collection method & Transition clinic & Control setting \\
\hline Semistructured interviews & $\begin{array}{l}5 \text { interviews: paediatric IBD nurse specialist } \\
(n=1) \text {, paediatric gastroenterologists }(n=2) \text {, } \\
\text { adult gastroenterologist }(n=1) \text { and one family } \\
\text { counsellor working in paediatric care }(n=1) \text {. }\end{array}$ & $\begin{array}{l}3 \text { interviews: paediatric gastroenterologist } \\
(n=1) \text {, adult care nurse }(n=1) \text { and adult } \\
\text { gastroenterologist }(n=1) \text {. }\end{array}$ \\
\hline Observations & $\begin{array}{l}3 \times 4 \text { hours of clinical consultations before } \\
\text { transfer. }\end{array}$ & $\begin{array}{l}2 \times 4 \text { hours of clinical consultations before } \\
\text { transfer. }\end{array}$ \\
\hline Surveys & $\begin{array}{l}n=21 \text { responded }(37.5 \%) ; n=14(25.0 \%) \text { filled out } \\
\text { the survey. }\end{array}$ & $\begin{array}{l}n=25 \text { responded }(46.3 \%) ; n=19(35.2 \%) \text { filled } \\
\text { out the survey. }\end{array}$ \\
\hline
\end{tabular}

IBD, inflammatory bowel disease.

\section{RESULTS}

\section{Study setting and response}

Two paediatric gastroenterology departments treating young people with IBD participated. One of these had implemented a TC in 2006. The other department had not (yet) implemented a transition programme or TC and served as control setting.

In both settings, interviews were held with professionals involved in transitional care. Three observations of 4 hours of outpatient consultations were performed at the TC and two in the control setting. At the TC, 56 young people had been transferred to adult care 2 to 4 years prior to data collection. Twenty-five of these $(44.6 \%)$ had been transferred to another hospital, implying that data after transfer were not readily available. In the control setting, the total study sample numbered 54, of whom $30(55.6 \%)$ had been transferred to another hospital. Of the total groups, 21 of the young people in the TC setting $(37.5 \%)$ and 25 of those in the control setting $(46.3 \%)$ responded to the invitation for the survey. Eventually, 14 (25\%) and 19 (35.2\%) filled out the survey, respectively. The study response is summarised in table 2. A description of the study sample is given in table 3 .

\section{Service structures and daily routines}

Joint delivery of outpatient care between paediatric and adult professionals was the main difference between the TC setting and the direct handover transfer setting (control setting). The TC was organised in an adult care setting; the multidisciplinary team consisted of two paediatric gastroenterologists, one adult gastroenterologist, one paediatric nurse specialist and one family counsellor working in paediatric care. In the direct handover care setting, only one paediatric gastroenterologist was

Table 3 Characteristics of the study samples of young people with IBD

\begin{tabular}{|c|c|c|c|}
\hline & $\begin{array}{l}\text { Transition clinic } \\
\text { ( } n=56 \text { before transfer; } \\
n=30 \text { after transfer) }\end{array}$ & $\begin{array}{l}\text { Control setting } \\
\text { ( } n=54 \text { before transfer; } \\
n=24 \text { after transfer) }\end{array}$ & P value \\
\hline Age at the time of survey (years) & $20.89( \pm 0.908)$ & $21.02( \pm 0.961)$ & 0.482 \\
\hline \multicolumn{4}{|l|}{ Diagnosis } \\
\hline IBD unclassified & $3(5.4 \%)$ & $6(11.1 \%)$ & \\
\hline Ulcerative proctitis & - & $1(1.9)$ & \\
\hline \multicolumn{4}{|l|}{ Timing of diagnosis } \\
\hline Within 2 years before transfer & $2(3.6 \%)$ & $11(20.4 \%)$ & 0.006 \\
\hline
\end{tabular}

*Independent samples t-test or Pearson $\chi^{2}$ test.

IBD, inflammatory bowel disease. 
involved in care before transfer. At the TC, young people aged 16 to 18 years were seen. They visited every 3 months before actual transfer to adult care. Three out of the four consultations per year were held with paediatric care professionals; the other consultation was with the adult care professional. Although this TC did not provide joint consultations, there was intensive collaboration and alignment between paediatric and adult providers (joint care). Prior to each TC, the paediatric and adult team together discussed the patients.

In both settings, during consultations attention was given to the medical aspects of IBD, such as dealing with the treatment and with treatment side effects. Young people's own ideas and responsibilities were also discussed in both settings, as well as the upcoming transfer. However, at the TC, more attention was given to non-medical topics, such as leisure, sports, independent living, work, family situation and psychological counselling, as well as differences between the paediatric and adult healthcare setting. Table 4 provides a summary of the differences in service structures, daily routines and content of consultations between the TC and control setting.

\section{Interview results}

Facilitators

The most important facilitators for the functioning of the TC are related to the healthcare team involved in transitional care. High engagement and motivation of healthcare professionals from both paediatric and adult care is essential according to all respondents at the TC. Paediatric and adult professionals should be willing to work together and perceive transitional care (and thus

Table 4 Differences in service structures and daily routines between the two settings

\begin{tabular}{|c|c|c|}
\hline Characteristics of care & TC setting & Control setting (direct handover care) \\
\hline Setting & Adult gastroenterology department & Paediatric gastroenterology department \\
\hline Team of professionals & $\begin{array}{l}\text { Paediatric IBD nurse specialist }(n=1) \text {, paediatric } \\
\text { gastroenterologists }(n=2) \text {, adult gastroenterologist }(n=1) \text { and one } \\
\text { family counsellor working in paediatric care }(n=1)\end{array}$ & Paediatric gastroenterologist $(\mathrm{n}=1)$ \\
\hline Visitors & $\begin{array}{l}\text { Young people with IBD aged } 16-18 \text { years with or without their } \\
\text { parents }\end{array}$ & $\begin{array}{l}\text { Young people with IBD with or without their parents } \\
\text { until age of } 18\end{array}$ \\
\hline Visits TC & Every 3 months & - \\
\hline $\begin{array}{l}\text { Follow-up after transfer } \\
\text { within the same hospital }\end{array}$ & $\begin{array}{l}\text { By an adult gastroenterologist, but not necessarily the one } \\
\text { involved at the TC }\end{array}$ & By an adult gastroenterologist or IBD nurse \\
\hline $\begin{array}{l}\text { Structure of care before } \\
\text { transfer }\end{array}$ & $\begin{array}{l}\text { 1. Each clinic starts with 1-hour multidisciplinary team meeting } \\
\text { of professionals, usually lead by the paediatric nurse } \\
\text { specialist } \\
\text { 2. Consultation with paediatric gastroenterologist or paediatric } \\
\text { nurse } \\
\text { 3. Consultation with adult gastroenterologist for selected young } \\
\text { people who will soon transfer to adult care; all young people } \\
\text { treated at the TC will see the adult care gastroenterologist } \\
\text { at least once before actual transfer to adult care, and } \\
\text { preferable once every year }\end{array}$ & $\begin{array}{l}\text { 1. No multidisciplinary team or nurse involved } \\
\text { 2. Measurements of weight and length by a medical } \\
\text { assistant } \\
\text { 3. Consultation with paediatric gastroenterologist }\end{array}$ \\
\hline
\end{tabular}

\begin{tabular}{|c|c|c|}
\hline $\begin{array}{l}\text { Topics discussed (content } \\
\text { of care) }\end{array}$ & $\begin{array}{l}\text { During team meeting: medical history and status, treatment and } \\
\text { lifestyle of patients (eg, smoking behaviour); when paediatric } \\
\text { nurse is in the lead extra topics are discussed: psychosocial } \\
\text { topics (social participation, family situation, psychological } \\
\text { counselling), treatment adherence, transfer to adult care (to } \\
\text { which department/hospital) } \\
\text { During consultation of paediatric healthcare professional } \\
\text { with young person (with or without parents): how is your IBD, } \\
\text { do you have complaints or symptoms you want to discuss, } \\
\text { which medication do you use (directed to patient), how is this } \\
\text { going, what are side effects, how is your eating behaviour, } \\
\text { smoking behaviour, limitations in daily living (sports, hobby's, } \\
\text { leisure, school, holidays), future plans, medication adherence, } \\
\text { transfer to adult care, explaining TC, importance of becoming } \\
\text { independent and own responsibility } \\
\text { During consultation of adult gastroenterologist with young } \\
\text { person (and/or parents): how is it going with the condition, } \\
\text { do you have complaints or symptoms you want to discuss, } \\
\text { which medication do you use (directed to patient), how is this } \\
\text { going, what are side effects, importance of own responsibility } \\
\text { (when taking medication), further treatment, explanation about } \\
\text { TC, differences between paediatric and adult care, choice of } \\
\text { department young person will be transferred to, activities of } \\
\text { young person (school, work, living) }\end{array}$ & $\begin{array}{l}\text { During consultation of paediatric gastroenterologist } \\
\text { with young person: physical examination by } \\
\text { healthcare professional, how is it going with the } \\
\text { condition, do you have complaints or symptoms } \\
\text { you want to discuss, how is it going with taking your } \\
\text { medication (how will you do this during holidays), } \\
\text { how are things going at school, school absenteeism, } \\
\text { further treatment (endoscopy and important of own } \\
\text { voice in healthcare from } 16 \text { years and older, including } \\
\text { transfer) }\end{array}$ \\
\hline
\end{tabular}

IBD, inflammatory bowel disease; TC, transition clinic. 
the TC) as important. The paediatric nurse explained: "What I noticed is that it is essential to have people who are enthusiastic, who think this is really important". The adult gastroenterologist seemed to agree: "You just need people who are enthusiastic and who can work together. We are doing this together". Another facilitator was the fact that the case load of patients seen at the TC was large enough for clinics to be structurally scheduled over the year.

\section{Barriers}

Barriers for the organisation of a TC were: (1) lack of service structure and alignment in content of care between paediatric and adult care, and little attention given to transition-specific topics; (2) time restrictions and planning difficulties; (3) limited involvement of professionals from adult care; (4) lack of financial coverage of the provided joint care. Professionals from both paediatric and adult care mentioned that they did not really coordinate who would discuss which topic with the young person (and his/her parents). Coordination could provide more structure in discussing important topics and preparing young persons for transition, but they feel that time is too short. The paediatric gastroenterologist explained: "So many things have to be done in so little time that often you don't find the time to address all important topics. [...] We need to bring more structure into the content discussed, because it can be valuable to discuss certain topics repeatedly". The adult gastroenterologist also explained: "Being involved in transitional care takes time, and that is always the big problem. We do not have time". In the control setting, time restrictions were an important reason for not organising a TC and the TC time restrictions were also the reason that only one dedicated adult gastroenterologist was involved at the TC: the young people did not get to know the other adult providers before transfer. Another important barrier was related to financial coverage. The adult gastroenterologist clarified: "The only reason [the TC] is organised is because both the paediatric gastroenterologist and I want to do this. We think this is important, but I think others don't find it as important as we do. No time and extra money are reserved for the TC. Although we provide joint care, my department doesn't get paid for this. I'm doing a lot of voluntary work and have done so for years now. Now I don't care, but my department doesn't get paid. That means that there is no incentive to do this". Financial support was also an issue according to the paediatric care professionals. A paediatric gastroenterologist explained: "The transition clinic is not financially supported by the hospital. All the extra work we do, is voluntary". Lack of reimbursement was also mentioned in the control setting as an important reason for not having a TC.

\section{Perceived benefits}

Professionals stated that creating patient awareness about the transition process, making patients feel more prepared for treatment in an adult department and making them feel more confident about the transfer and self-management were the most important assets of a TC for young people. Parents could benefit from increased awareness about transition and feeling supported to help their children to become more independent. Professionals themselves benefitted because the TC encouraged them to work and learn together. By aligning treatment protocols across paediatric and adult care departments, quality of care was enhanced. Professionals thought the young people would feel safer because of this collaboration. A paediatric gastroenterologist added: "We also want the transition to go smoothly. We do not want our patients to get sicker after transfer. We want things to go well and want them to feel confident and safe".

\section{Differences in healthcare use}

The young people who received care at the TC on average had significantly more planned consultations in the year before transfer than those in direct handover care (table 5). As for missed consultations, those who received care at the TC had significantly fewer missed consultations in the second year after transfer. Over the whole period, young people treated at the TC had significantly fewer planned and unplanned hospital admissions related to IBD. For young people at the TC, the department and hospital they had been transferred to was more often recorded in the patient chart compared with those in direct handover care.

\section{Differences in clinical measures}

Significantly more young people in direct handover care had clinically active disease during transfer compared with those seen at the TC (table 5). The young people in direct handover care also more often experienced a relapse in the year before transfer. Also, the use of medications differed significantly between both groups. In the direct handover group, more young people used biologics compared with the TC group. This difference was found at all data collection points.

\section{Transition experiences and satisfaction with transfer}

Regarding transition experiences, young people treated at the TC were more positive on whether their adult healthcare providers were familiar with their personal situation and whether they had met their adult healthcare provider(s) before transfer (table 6). Overall, the young people treated at the TC tended to report higher scores for transition experiences compared with those treated in direct handover care. This difference was not statistically significant, but the effect size was medium. The same was the case for reported satisfaction with the transfer.

\section{Healthcare-related and self-management-related outcomes (at the time of survey)}

The perceived patient-centredness score was slightly higher at the TC, as was the case for self-efficacy, adherence to treatment, self-management and health-related quality of life (table 6). On the other hand, young people in the direct handover care setting reported a slightly 
Table 5 Clinical and healthcare use outcomes of young people with IBD

\section{Chart review results}

TC

Control setting

Effect size*

P value†

No of charts reviewed $\ddagger$

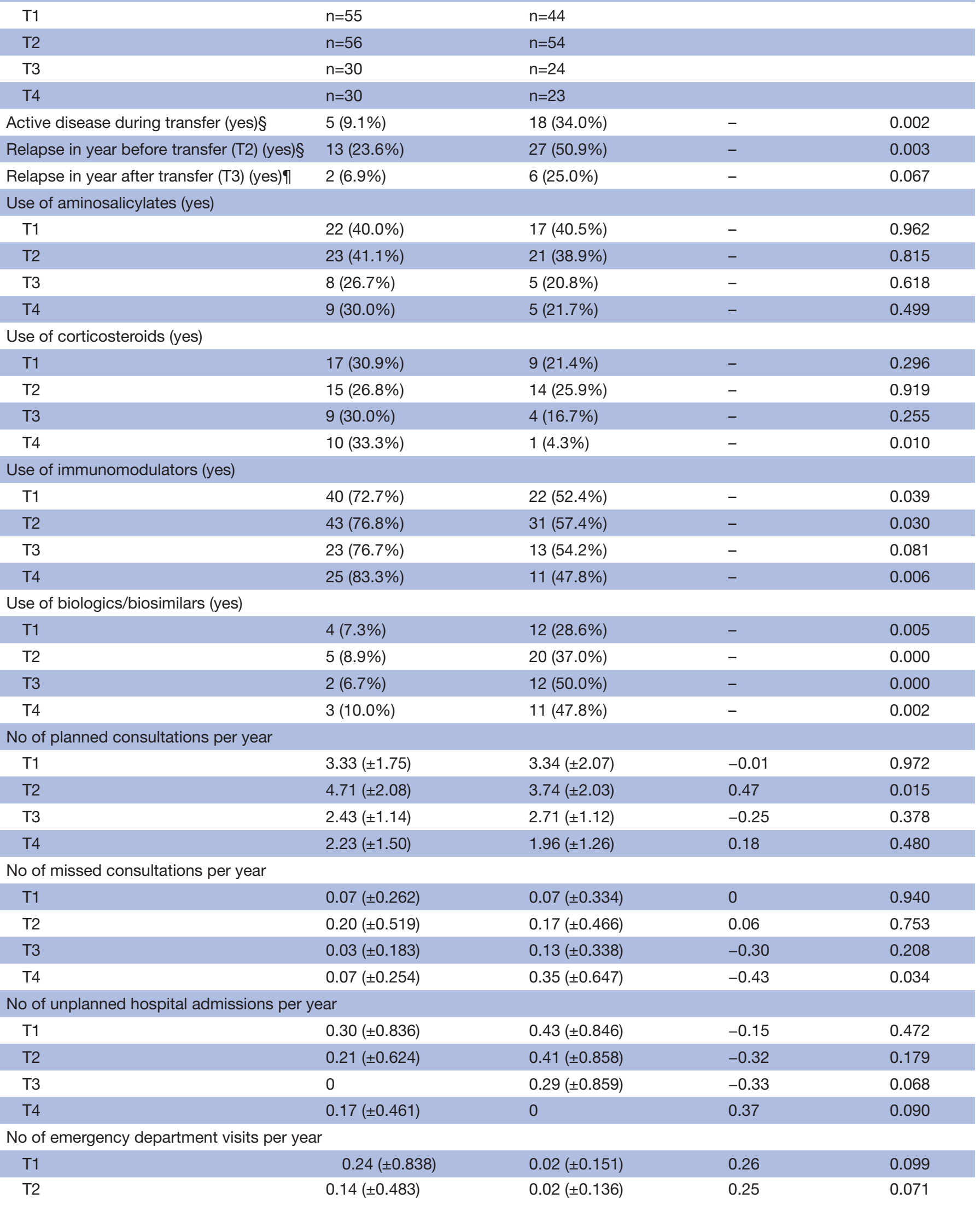




\begin{tabular}{|c|c|c|c|c|}
\hline Chart review results & TC & Control setting & Effect size* & $\mathbf{P}$ value \\
\hline T3 & $0.03( \pm 0.183)$ & $0.25( \pm 0.847)$ & 0.26 & 0.178 \\
\hline $\mathrm{T} 4$ & 0 & 0 & - & - \\
\hline $\begin{array}{l}\text { Department to which young person } \\
\text { transferred is recorded/known (yes) }\end{array}$ & $55(98.2 \%)$ & $48(88.9 \%)$ & - & 0.045 \\
\hline
\end{tabular}

${ }^{*}$ Cohen's d (based on largest SD).

†Independent samples t-test or Pearson $\chi^{2}$ test.

$\ddagger$ Second year before transfer=T1, year before transfer=T2, year after transfer=T3, second year after transfer=T4.

§Control setting $n=53$, TC setting $n=55$.

ITC setting $n=29$.

IBD, inflammatory bowel disease; TC, transition clinic.

higher score on independence during consultations. However, none of the differences in healthcare-related and self-management-related outcomes was statistically significant and effect sizes were small.

\section{DISCUSSION}

Unlike to the direct handover care setting, the evaluated outpatient TC for young people with IBD conducted multidisciplinary team meetings of professionals of both paediatric and adult care, as well as consultations between young people and adult care professionals before transfer. Interestingly, we found positive trends in young people's satisfaction and experiences with the transfer when treated at the TC, suggesting that the TC may foster the quality of transitional care. However, the differences found were not statistically significant (likely due to the low response rates in the survey study). Also, literature suggests that young people prefer professionals of both paediatric and adult care be present at the same time in consultations (joint consultations). ${ }^{25}$ The evaluated TC provided separate consultations, however, and providing joint consultations may possibly elicit more positive experiences.

In the recent topical review by the Paediatric Committee of the European Crohn's and Colitis Organisation (P-ECCO), the importance was emphasised of empowering young people by nurturing their knowledge and skills to manage IBD. ${ }^{3}$ Participation in a transition programme should enable this and specific interventions are useful to do so. ${ }^{32627}$ Examples of interventions are seeing young people alone, without parents, during consultations or preparing an individual transition

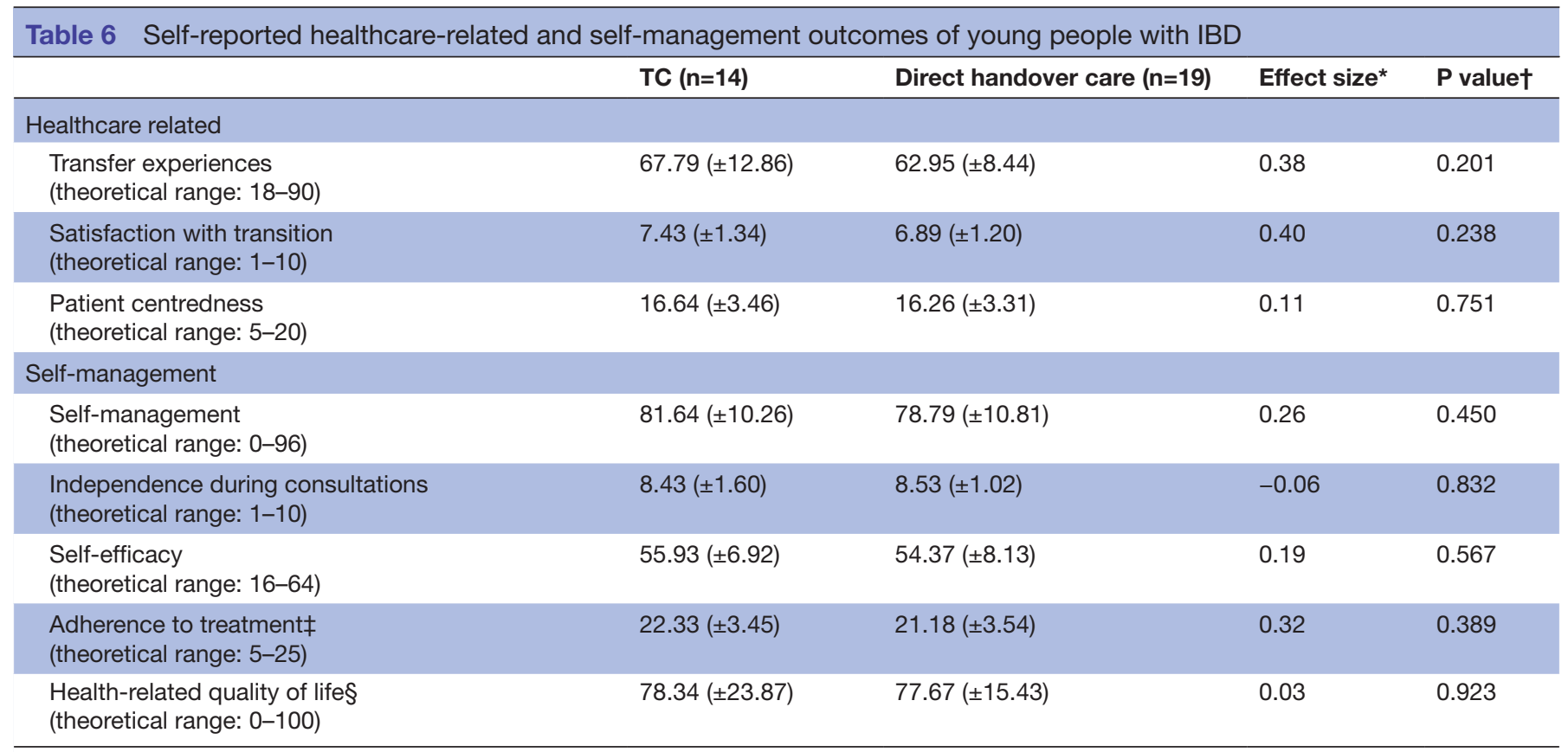

${ }^{*}$ Cohen's d (based on largest SD).

†Independent samples t-test.

†TC group: $n=12$; direct handover care group: $n=17$.

$\S$ Direct handover care group: $n=18$.

IBD, inflammatory bowel disease; TC, transition clinic. 
plan. ${ }^{28}$ However, the TC we evaluated did not provide structural interventions or transition plans such as Ready Steady Go. ${ }^{29}$ In both settings, there was no policy for split consultations and young people were not routinely seen on their own. Professionals recognised the need for more structure in content of the process and more attention for transition-specific topics. Mere awareness of the importance of transition among healthcare providers is not enough to foster young people's skills. ${ }^{30}$ This could help explain why no significant differences were found in selfmanagement-related experiences between young people treated at the TC and those treated at the control setting, although another study on a TC for young people with IBD did find significant positive effects on self-efficacy. ${ }^{31}$ Still, the low response rate and possible recall bias of young people who transferred over 2 years ago could also be held responsible for this.

Young people treated at the TC had fewer missed consultations after transfer than those treated at the control setting. This finding is in line with current literature where strong evidence is found for the enhancement of clinic attendance ${ }^{1}$ and this topic is also mentioned in the practice points of the topical review of P-ECCO. ${ }^{3}$ A positive effect of TCs on the continuity and safety of gastroenterology care has been reported previously. ${ }^{32}$ Another study of outcomes in adolescents with IBD following transfer also found fewer missed consultations in the group that received transitional care. ${ }^{33}$ In our study, young people treated at the direct handover setting more often had a relapse in the year before transfer and were more often transferred while dealing with clinically active disease. At the TC, specific attention was given to disease activity, since it is known that having active disease at transfer can negatively influence the transition process. ${ }^{34}$ The professionals preferred to postpone the transfer until the disease was in clinical remission. This flexibility of timing of transfer is recommended for direct handover IBD care too.

Important barriers for the organisation of a TC were time restrictions and planning difficulties, lack of financial coverage and a lack of awareness of the importance of transitional care among adult care providers. In the literature, these issues are also described as barriers to successful transition for young people. ${ }^{11}$ This finding highlights the need to embed transitional care in healthcare policy, so that time and resources can be made available to provide good transitional care. Recent and current development of guidelines and consensus statements suggest that this awareness is growing in the field. However, the next question is how awareness may be translated to actual healthcare policy measures and how these recommendations can be implemented in daily healthcare practice.

\section{CONCLUSION}

This evaluation of a TC demonstrated that young people with IBD who had been treated at a TC had fewer missed consultations after transfer than those treated in direct handover care. They were less likely to have active disease during transfer, less often experienced a relapse in the year before transfer and showed a positive trend for experiences and satisfaction with transfer. Still, no significant differences were found in self-management-related experiences of young people with IBD treated at the TC compared with those in the control setting, suggesting there is room for improvement in the TC model. This was also suggested by the qualitative results of the study; that is, healthcare providers mentioned several organisational and financial challenges that may have influenced the outcomes. Although there are developments in guidelines and consensus statements about the organisation and content of transitional care, the next step is to implement these recommendations in healthcare policy and daily practice.

Acknowledgements The authors thank Martha van Gaalen, nurse specialist at Erasmus MC-Sophia Children's Hospital, who assisted in the study logistics of the study at participating department.

Contributors JNTS: conception and design of the study, acquisition of data, analysis and interpretation of data, drafting the article, revision of the article and final approval of the version submitted. MACP: acquisition of data, analysis and interpretation of the data, revising the article critically for important intellectual content and final approval of the version to be submitted.JH: acquisition of data, revising the article critically for important intellectual content and final approval of the version to be submitted. AvS: conception and design of the study, interpretation of the data, revising the article critically for important intellectual content and final approval of the version to be submitted.VMW: acquisition and interpretation of data revising the article critically for important intellectual content and final approval of the version to be submitted.JCE: design of the study, acquisition and interpretation of data, revising the article critically for important intellectual content and final approval of the version to be submitted.

Funding This work was supported by Foundation Innovation Alliance (SIA-RAAK) with funding from the Dutch Ministry of Education, Culture and Science (Grant no: PR0-02-025). The funding organisation had no role in the design, implementation, interpretation and reporting of this study.

Competing interests JCE received research support from MSD and has served as advisory board member for Janssen and Abbvie. AvS has served as advisory board member for professional education in diabetes care for Medtronic.

Patient consent for publication Not required.

Ethics approval The Medical Ethical Committees of the Erasmus Medical Center (MEC-2014-246) and University Medical Center Utrecht (METC-15-123/C) approved the study protocol.

Provenance and peer review Not commissioned; externally peer reviewed. Data availability statement No data are available.

Open access This is an open access article distributed in accordance with the Creative Commons Attribution Non Commercial (CC BY-NC 4.0) license, which permits others to distribute, remix, adapt, build upon this work non-commercially, and license their derivative works on different terms, provided the original work is properly cited, appropriate credit is given, any changes made indicated, and the use is non-commercial. See: http://creativecommons.org/licenses/by-nc/4.0/.

\section{ORCID iD}

Jane N T Sattoe http://orcid.org/0000-0002-0433-5022

\section{REFERENCES}

1 Brooks AJ, Smith PJ, Cohen R, et al. Uk guideline on transition of adolescent and young persons with chronic digestive diseases from paediatric to adult care. Gut 2017;66:988-1000.

2 Philpott JR, Kurowski JA. Challenges in transitional care in inflammatory bowel disease: a review of the current literature 
in transition readiness and outcomes. Inflamm Bowel Dis 2019;25:45-55.

3 van Rheenen PF, Aloi M, Biron IA, et al. European Crohn's and colitis organisation topical review on transitional care in inflammatory bowel disease. J Crohns Colitis 2017;11:1032-8.

4 Elli L, Maieron R, Martelossi S, et al. Transition of gastroenterological patients from paediatric to adult care: a position statement by the Italian societies of gastroenterology. Dig Liver Dis 2015;47:734-40.

5 Lindfred H, Saalman R, Nilsson S, et al. Self-reported health, selfmanagement, and the impact of living with inflammatory bowel disease during adolescence. J Pediatr Nurs 2012;27:256-64.

6 Goodhand J, Hedin CR, Croft NM, et al. Adolescents with IBD: the importance of structured transition care. J Crohns Colitis 2011;5:509-19.

7 Brooks AJ, Rowse G, Ryder A, et al. Systematic review: psychological morbidity in young people with inflammatory bowel disease - risk factors and impacts. Aliment Pharmacol Ther 2016;44:3-15

8 Kemp K, Griffiths J, Lovell K. Understanding the health and social care needs of people living with IBD: a meta-synthesis of the evidence. World J Gastroenterol 2012;18.

9 Bishop J, Lemberg DA, Day A. Managing inflammatory bowe disease in adolescent patients. Adolesc Health Med Ther 2014;5:1-13.

10 Fegran L, Hall EOC, Uhrenfeldt L, et al. Adolescents' and young adults' transition experiences when transferring from paediatric to adult care: a qualitative metasynthesis. Int J Nurs Stud 2014;51:123-35

11 Clarke T, Lusher J. Transitioning patients with inflammatory bowel disease (IBD) from adolescent to adult services: a systematic review. Frontline Gastroenterol 2016:7:264-70.

12 Mazur A, Dembinski L, Schrier L, et al. European Academy of paediatric consensus statement on successful transition from paediatric to adult care for adolescents with chronic conditions. Acta Paediatr 2017;106:1354-7.

13 Crowley R, Wolfe I, Lock K, et al. Improving the transition between paediatric and adult healthcare: a systematic review. Arch Dis Child 2011;96:548-53.

14 Carrizosa J, An I, Appleton R, et al. Models for transition clinics. Epilepsia 2014;55 Suppl 3:46-51.

15 Gray WN, Maddux MH. Current transition practices in pediatric IBD: findings from a national survey of pediatric providers. Inflamm Bowel Dis 2016;22:372-9.

16 Sattoe JNT, Peeters MAC, Hilberink SR, et al. Evaluating outpatient transition clinics: a mixed-methods study protocol. BMJ Open 2016;6:e011926.

17 Hargreaves DS. Revised you're welcome criteria and future developments in adolescent healthcare. J Clin Res Pediatr Endocrinol 2011;3:43-50.

18 Shaw KL, Southwood TR, McDonagh JE, et al. Development and preliminary validation of the ?Mind the Gap? scale to assess satisfaction with transitional health care among adolescents with juvenile idiopathic arthritis. Child Care Health Dev 2007;33:380-8.

19 van Staa AL, Jedeloo S, van Meeteren J, et al. Crossing the transition chasm: experiences and recommendations for improving transitional care of young adults, parents and providers. Child Care Health Dev 2011;37:821-32.

20 Lugasi T, Achille M, Stevenson M. Patients' perspective on factors that facilitate transition from child-centered to adult-centered health care: a theory integrated metasummary of quantitative and qualitative studies. J Adolesc Health 2011;48:429-40.

21 Betz CL, Lobo ML, Nehring WM, et al. Voices not heard: a systematic review of adolescents' and emerging adults' perspectives of health care transition. Nurs Outlook 2013:61:311-36.
22 Sonneveld HM, Strating MMH, van Staa AL, et al. Gaps in transitional care: what are the perceptions of adolescents, parents and providers? Child Care Health Dev 2013;39:69-80.

23 Nehring WM, Betz CL, Lobo ML. Uncharted Territory: systematic review of providers' roles, understanding, and views pertaining to health care transition. J Pediatr Nurs 2015;30:732-47.

24 Suris J-C, Akre C. Key elements for, and indicators of, a successful transition: an international Delphi study. J Adolesc Health 2015;56:612-8.

25 Bennett AL, Moore D, Bampton PA, et al. Outcomes and patients perspectives of transition from paediatric to adult care in inflammatory bowel disease. World J Gastroenterol 2016;22.

26 Brooks AJ, Smith PJ, Lindsay JO. Monitoring adolescents and young people with inflammatory bowel disease during transition to adult healthcare. Frontline Gastroenterol 2018;9:37-44.

27 Carlsen K, Haddad N, Gordon J, et al. Self-efficacy and resilience are useful predictors of transition readiness scores in adolescents with inflammatory bowel diseases. Inflamm Bowel Dis 2017;23:341-6.

28 van Staa A, Sattoe JNT, Strating MMH. Experiences with and outcomes of two interventions to maximize engagement of chronically ill adolescents during hospital consultations: a mixed methods study. J Pediatr Nurs 2015;30:757-75.

29 Nagra A, McGinnity PM, Davis N, et al. Implementing transition: ready steady go. Arch Dis Child Educ Pract Ed 2015;100:313-20.

30 Fishman LN, Ziniel SI, Adrichem ME, et al. Provider awareness alone does not improve transition readiness skills in adolescent patients with inflammatory bowel disease. J Pediatr Gastroenterol Nutr 2014;59:221-4.

31 Yerushalmy-Feler A, Ron Y, Barnea E, et al. Adolescent transition clinic in inflammatory bowel disease: quantitative assessment of selfefficacy skills. Eur J Gastroenterol Hepatol 2017;29:831-7.

32 Little R, McLoughlin L, Szabo A. 9 transforming transition for paediatric IBD patients in Northern Ireland. Gut 2017;66:A4.

33 Cole R, Ashok D, Razack A, et al. Evaluation of outcomes in adolescent inflammatory bowel disease patients following transfer from pediatric to adult health care services: case for transition. J Adolesc Health 2015;57:212-7.

34 van den Brink G, van Gaalen MAC, Zijlstra M, et al. Self-efficacy did not predict the outcome of the transition to adult care in adolescents with inflammatory bowel disease. Acta Paediatr 2019;108:333-8.

35 van Staa A, Sattoe JNT. Young adults' experiences and satisfaction with the transfer of care. J Adolesc Health 2014:55:796-803.

36 Arah OA, ten Asbroek AHA, Delnoij DMJ, et al. Psychometric properties of the Dutch version of the hospital-level consumer assessment of health plans survey instrument. Health Serv Res 2006;41:284-301.

37 W Battersby M, Ask A, M Reece M, et al. The partners in health scale: the development and psychometric properties of a generic assessment scale for chronic condition self-management. Aust $J$ Prim Health 2003;9:41-52.

38 Petkov J, Harvey P, Battersby M. The internal consistency and construct validity of the partners in health scale: validation of a patient rated chronic condition self-management measure. Qual Life Res 2010;19:1079-85.

39 van Staa A. On your own feet: preferences and competencies for care of adolescents with chronic conditions, in Erasmus School of Health Policy \& Management. Erasmus University Rotterdam: Rotterdam, 2012

40 Mahler C, Hermann K, Horne R, et al. Assessing reported adherence to pharmacological treatment recommendations. translation and evaluation of the medication adherence report scale (MARS) in Germany. J Eval Clin Pract 2010;353:574-9.

41 Limperg PF, Haverman L, van Oers HA, et al. Health related quality of life in Dutch young adults: psychometric properties of the PedsQL generic core scales young adult version. Health Qual Life Outcomes 2014;12:9. 